\title{
Marketing estratégico en redes sociales de las organizaciones. Un abordaje $360^{\circ}$
}

\section{Strategic marketing in organization's social networks. An approach $360^{\circ}$}

\author{
Mg. Diego Bonilla-Jurado ${ }^{1}$ \\ administracion@bhconsultores.com \\ Psc Ind. Diego Salinas-Morales ${ }^{2}$ \\ talentohumano@bhconsultores.com \\ Ing. Freddy Lalaleo-Analuisa ${ }^{3}$ \\ produccion@bhconsultores.com \\ Mg. Santiago Velastegui-Hernández \\ rs.velastegui@uta.edu.ec
}

Recibido: 1/09/2017, Aceptado: 1/11/2017

\section{RESUMEN}

El marketing en redes sociales, o mejor conocido como Social Media Marketing se ha convertido en un elemento integral en los negocios de las organizaciones del siglo XXI. Sin embargo, la literatura acerca de este tema continúa fragmentada y se centra en cuestiones aisladas (un ejemplo claro, el abordaje a las tácticas para una comunicación efectiva). El actual desarrollo aplica un enfoque cualitativo mediante la construcción de teorías para el desarrollo de un marco estratégico que cohesiona cuatro dimensiones genéricas de marketing estratégico de medios sociales: medios de marketing social que defienden a los "exploradores", medios sociales y la cultura de marketing donde se incluye a los polos del conservadurismo y el modernismo, los medios de comunicación social y las estructuras de comercialización de las jerarquías y redes, y social media marketing de la autocracia a la anarquía. Al proporcionar una conceptualización integral y una definición de marketing en medios sociales estratégicos, esta compilación propone un marco integrador que se expande más allá de la teoría de marketing existente. Además, los administradores pueden aplicar dicho marco para posicionar sus organizaciones en estas cuatro dimensiones de una manera consistente mostrando el cumplimiento de su misión de empresa y sus objetivos.

\footnotetext{
${ }^{1}$ BH Consultores. Ecuador

${ }^{2}$ BH Consultores. Ecuador

${ }^{3} \mathrm{BH}$ Consultores. Ecuador

${ }^{4}$ Docente de la Universidad Técnica de Ambato-Ecuador
}

Revista científica Ciencia y Tecnología Vol 18 No 17 págs. 72-83 http://cienciaytecnologia.uteg.edu.ec 


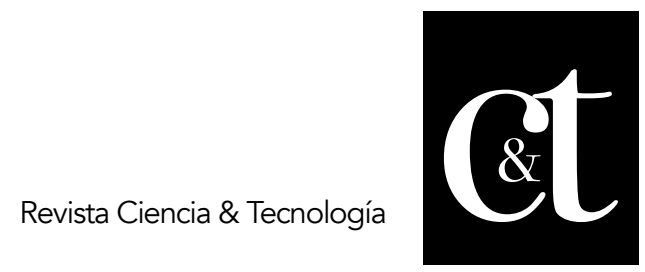

No. 17, 31 de enero de 2018

ISSN impreso: 1390 - 6321

Palabras clave: Marketing estratégico, social media marketing, marketing en redes sociales, publicidad digital

\begin{abstract}
Social media marketing, or better known as Social Media Marketing, has become an integral element in 21 st century business. However, the literature on this current topic remains fragmented and focuses on specific issues (a clear example, the approach of tactics for effective communication). The current development applies a qualitative approach by building technologies for the strategic development of social media: social marketing media that define the "explorers", social media and marketing culture that includes the poles of conservatism and modernism, social media and marketing structures of hierarchies and networks, and social media marketing from autocracy to anarchy. By providing a comprehensive conceptualization and definition of marketing in strategic social media, this compilation proposes an integrating framework that expands beyond the existing marketing theory. In addition, customers can apply this framework to position their organizations in these four dimensions in a consistent manner showing the fulfillment of their company mission and objectives.
\end{abstract}

Keywords: Strategic marketing, social media marketing, digital advertising

\title{
Introducción
}

La comprensión del papel de los medios sociales en el contexto de la comercialización es fundamental (por ejemplo, Fong \& Burton, 2008; Kumar, Bezawada, Rishika, Janakiraman, \& Kannan, 2016; Schultz \& Peltier, 2013). La mayoría de los estudios existentes se centran en cuestiones particulares como comportamiento de compra (Chang, Yu, y Lu, 2015; Kumar et al, 2016.; Relling, Schnittka, Sattler, \& Johnen, 2016), la gestión de relaciones con los clientes (Trainor, Andzulis, Rapp, y Agnihotri, 2014), la gestión de la marca (Asmussen, Harridge-March, Occhiocupo, \& Farquhar, 2013), la gestión de la innovación (Gebauer, Fuller, \& Pezzei, 2013), y la contratación de empleados (Sivertzen, Nilsen, \& Olafsen, 2013). Si bien existen avances en áreas especializadas de medios de comunicación social en un contexto de marketing y gestión, la literatura existente no proporciona un marco integral para el marketing en medios sociales a nivel estratégico. Dicha deficiencia es sorprendente porque los académicos (Labrecque, vor dem Esche, Mathwick, Novak, \& Hofacker, 2013; Schultz \& Peltier, 2013; Yadav \& Pavlou, 2014) y profesionales (Divol, Edelman, \& Sarrazin, 2012) reconocen las nuevas complejidades que acompañan a estos medios de comunicación, estando de acuerdo en que la investigación del marketing en medios sociales necesita una reconceptualización. En otras palabras, el marketing estratégico de medios sociales sigue siendo un paradigma de interacción del usuario no probado (Naylor, Lamberton, \& West, 2012) con poca investigación académica publicada.

El presente desarrollo tiene como objetivo abordar la brecha de investigación teórica y gerencialmente importante para las organizaciones mediante la exploración de las siguientes dos preguntas de investigación: ¿Cómo se define y conceptualiza el marketing estratégico en redes sociales? y ¿Qué factores exigen consideración al construir una estrategia de marketing en redes sociales de una organización? específicamente, esta documento intenta definir los continuos en los que se basan las decisiones estratégicas de marketing en redes sociales e integrarlas en un marco holístico. 


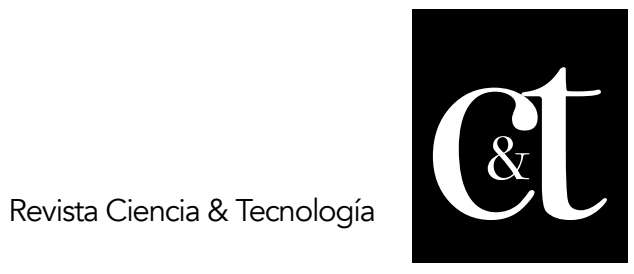

No. 17, 31 de enero de 2018

ISSN impreso: 1390 - 6321

El aporte teórico de este desarrollo es triple. En primer lugar, esta investigación ofrece una amplia definición y conceptualización de marketing estratégico de medios sociales. Esta conceptualización va más allá de un enfoque aislado en los consumidores $\mathrm{y} / \mathrm{o}$ aspectos comunicativos discutidos en la literatura existente de marketing en redes sociales. Hallazgos, marcos de trabajo y teorías de disciplinas como recursos humanos (Sivertzen et al., 2013), gestión organizacional (Baird \& Parasnis, 2013), Relaciones públicas (Eyrich, Padman, \& Sweetser, 2008), y comunicaciones (Linke \& Zerfass, 2013) representan importantes elementos del marketing estratégico de medios sociales. En segundo lugar, se define un marco teórico que describe las dimensiones cruciales en las que se desarrollan las redes sociales estratégicas, las decisiones, y las ventajas y desventajas involucradas en el posicionamiento de una empresa a lo largo de cada uno de los procesos claves. En tercer lugar, este desarrollo se integra al marketing en medios sociales en un contexto de marketing y gestión más estratégico, generando una comprensión teórica más avanzada acerca del tema, proporcionando una guía para la toma de decisiones gerenciales.

\section{Desarrollo}

\section{Objetivos y resultados de marketing en redes sociales}

Las aportaciones existentes investigan empíricamente los objetivos específicos de comercialización de redes sociales (por ejemplo, Ashley y Tuten, 2015; Bernoff y Li, 2008; Bianchi y Andrews, 2015; Schultz y Peltier, 2013), donde se incluye estimular las ventas, aumentar el conocimiento de la marca, mejorar la imagen de marca, generar tráfico a las plataformas en línea, reducir los costos de comercialización y crear interacción con los usuarios en las plataformas al estimular a los mismos a publicar o compartir contenido. Junto con estos objetivos predominantemente proactivos, las empresas pueden utilizar el marketing social de medios de una manera más reactiva. Por ejemplo, las empresas pueden monitorear y analizar conversaciones en las redes sociales para comprender cómo los consumidores ven una empresa o sus acciones (Schweidel y Moe, 2014). Muchas empresas también intentan reducir los riesgos del uso indebido de las redes sociales por parte de sus empleados estableciendo reglas sobre cómo las redes sociales deberían usarse en contextos relacionados con el trabajo (Rokka, Karlsson, y Tienari, 2014). De hecho, los objetivos y desafíos específicos de marketing en redes sociales pueden depender de factores como la industria y el tamaño de la empresa.

Las condiciones del entorno de la comercialización eficaz del social media

El desarrollo también expone los elementos fundamentales que constituyen las redes sociales para comprender mejor las potenciales implicaciones positivas y negativas para la empresa (Hennig-Thurau et al., 2010; Labrecque et al., 2013). Por ejemplo, Kietzmann, Hermkens, McCarthy, y Silvestre (2011) identifican siete componentes funcionales comunes en todas las formas de redes sociales: identidad, conversación, intercambio, presencia, relaciones, reputación y grupos. Sin embargo, los vendedores de redes sociales utilizan estos elementos con diferentes énfasis para crear valor para los usuarios.

La efectividad del marketing en redes sociales también puede depender del papel específico que los consumidores asignan a las empresas y marcas dentro de la esfera de los medios sociales. Los consumidores pueden percibir a las compañías y marcas como "intrusos", "colados" (Fournier y Avery, 2011), o no deseados huéspedes en el 


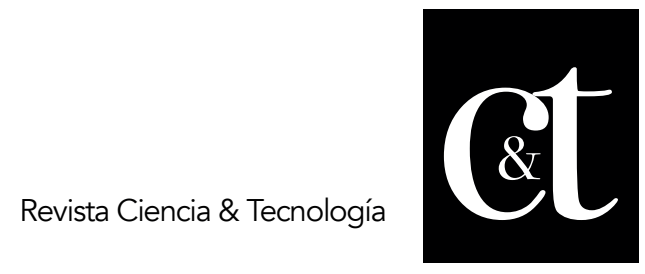

No. 17, 31 de enero de 2018

ISSN impreso: 1390 - 6321

espacio interactivo (Schultz y Peltier, 2013). Por el contrario, Ashley y Tuten (2015), Canhoto y Clark (2013) sugieren que muchos usuarios esperan que las empresas participen en las redes sociales e incluso pueden empujar deliberadamente a las empresas a la conversación en las redes sociales al mencionar la marca o al "etiquetado" de la empresa. Esta discrepancia puede reflejar una base heterogénea de consumidores, en la que un grupo de consumidores se siente cómodo con firmas proactivas y comprometidas, mientras que otra rechaza la invasión corporativa del espacio de las redes sociales.

Finalmente, las investigaciones mencionan que tanto el tipo de industria como el tipo de producto influyen en el impacto del marketing en redes sociales. En resumen, la literatura de mercadotecnia identifica los factores que influyen en la efectividad de los esfuerzos de mercadotecnia en los medios sociales. Entonces, por lo tanto, esto se convierte en un marco de comercialización que va más allá de las características específicas de la empresa o del mercado para mostrar las dimensiones generales en las que los gerentes toman decisiones de marketing de redes sociales firmes 0 situacionales específicas.

La necesidad de un enfoque holístico del marketing en redes sociales

Desarrollos actuales no analizan al social media marketing de una forma holística como el presente desarrollo, refiriéndose a la idea de que los componentes de un todo no pueden separarse, Brunner-Sperdin, Scholl-Grissemann, y Stokburger-Sauer (2014), definen al término como la configuración de los componentes que determinan en última instancia una respuesta a una situación, opción o concepto. La interconectividad y la complejidad de las plataformas de medios sociales hacen que la gestión, incluso la conceptualización de la responsabilidad empleada con respecto a estas tecnologías es extremadamente complicada. Por ejemplo, Rokka et al. (2014), evidencian que el manejo, capital humano y clientes que operan y componen las redes sociales constituyen un significado diferente. Los mencionados autores concluyen que sus investigaciones trabajaban hacía, pero no habían desarrollado claramente, un marco para gestionar las responsabilidades de sus empleados dentro de la esfera de los medios sociales.

Quinton (2013) propone un movimiento desde una perspectiva relacional para una orientación interactiva centrándose en las interacciones de múltiples capas que pueden cruzar ambos ámbitos y medios de comunicación. Mientras que la orientación relacional se enfoca en la comunicación, la orientación interaccional pone énfasis en las relaciones multifacéticas basadas en el reparto dentro y entre las comunidades habilitadas digitalmente (Thompson \& Coskuner-Balli, 2007). Por lo tanto, en una expansión similar a la transformación de la publicidad a la comunicación integrada de marketing, los vendedores de medios sociales deben ser conscientes de la gran cantidad de posibles restricciones y consecuencias derivadas de la participación en los diferentes lugares de los medios sociales.

De Swaan Arons, van de Driest, \& Weed (2014) realzan el dinamismo extremo del marketing en la era digital. Se remarca que herramientas y estrategias que eran de vanguardia hace tan sólo unos años se están convirtiendo rápidamente en obsoletas, y los nuevos enfoques están apareciendo cada día. Por lo tanto, los vendedores hombres de mediana edad deben adaptarse a nuevos desafíos, junto con cambios en la organización y nuevas filosofías. El ejemplo claro se evidencia en la inclusión de 


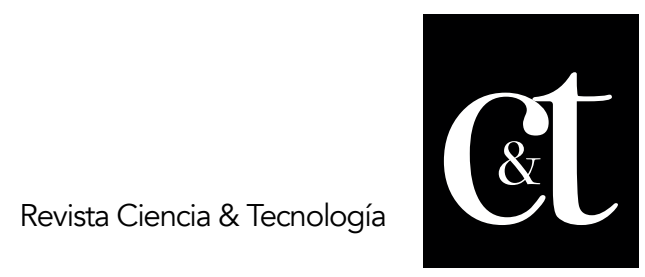

No. 17, 31 de enero de 2018

ISSN impreso: 1390 - 6321

otros departamentos o empleados en acciones de marketing de forma continua. De Swaan Arons et al. (2014, p. 59) concluyen que el marketing se ha convertido en un asunto demasiado importante para ser designado sólo a los vendedores. Por último, la literatura de marketing no aborda otros retos asociados a los medios sociales, como las responsabilidades de los vendedores (por ejemplo, los empleados que se encargan de la gestión de marketing en redes sociales) y el papel de los grupos de interés internos y externos (por ejemplo, normas y recomendaciones para el gobierno de la presencia en los medios sociales). Por lo tanto, la percepción de percibir la necesidad de un marco holístico e interdisciplinario para la conceptualización de marketing estratégico de medios sociales es primordial.

Marco estratégico del social media marketing

Los estudios realizados por Felix et al. (2016), nos muestran como resultado un marco estratégico del social media marketing, compuesto de cuatro dimensiones centrales. Como primera dimensión, se muestra la divergencia entre "defensores" y "exploradores" acerca de este tema, quienes emplean esta herramienta de forma diferente, como medio de comunicación unidireccional solamente para las partes interesadas (defensores), o la emplean como medio de interacción entre partes recíprocas y diferentes tales como clientes, empleados, proveedores y agentes de gobierno (exploradores). El segundo punto distingue al conservadurismo, que se encuentra representado por un enfoque encapsulado y tradicional, y al modernismo, que se caracteriza por un enfoque más permeable, abierto, con medios de comunicación social asociados a una cultura flexible. El tercer punto diferencia a las jerarquías, representada de forma evidente por la clara centralización de las actividades en una persona específica, y a las redes quienes representan una estructura organizacional en donde todos los empleados son responsable del manejo del marketing en redes sociales, dejando de lado la posibilidad y necesidad de un director de marketing específico en sus filas. En cuarto lugar, se evidencia a la autocracia, la cual permite a la empresa interactuar en diferentes plataformas de medios sociales con la ayuda de regulaciones y normas, y a la anarquía, la cual crea situaciones que no permiten a la organización moverse de forma libre en función de ciertas directrices y normas de orden gubernamental. 


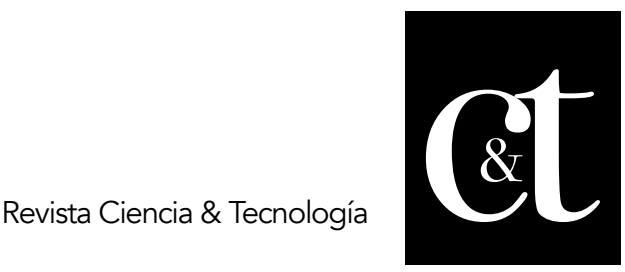

No. 17, 31 de enero de 2018

ISSN impreso: 1390 - 6321

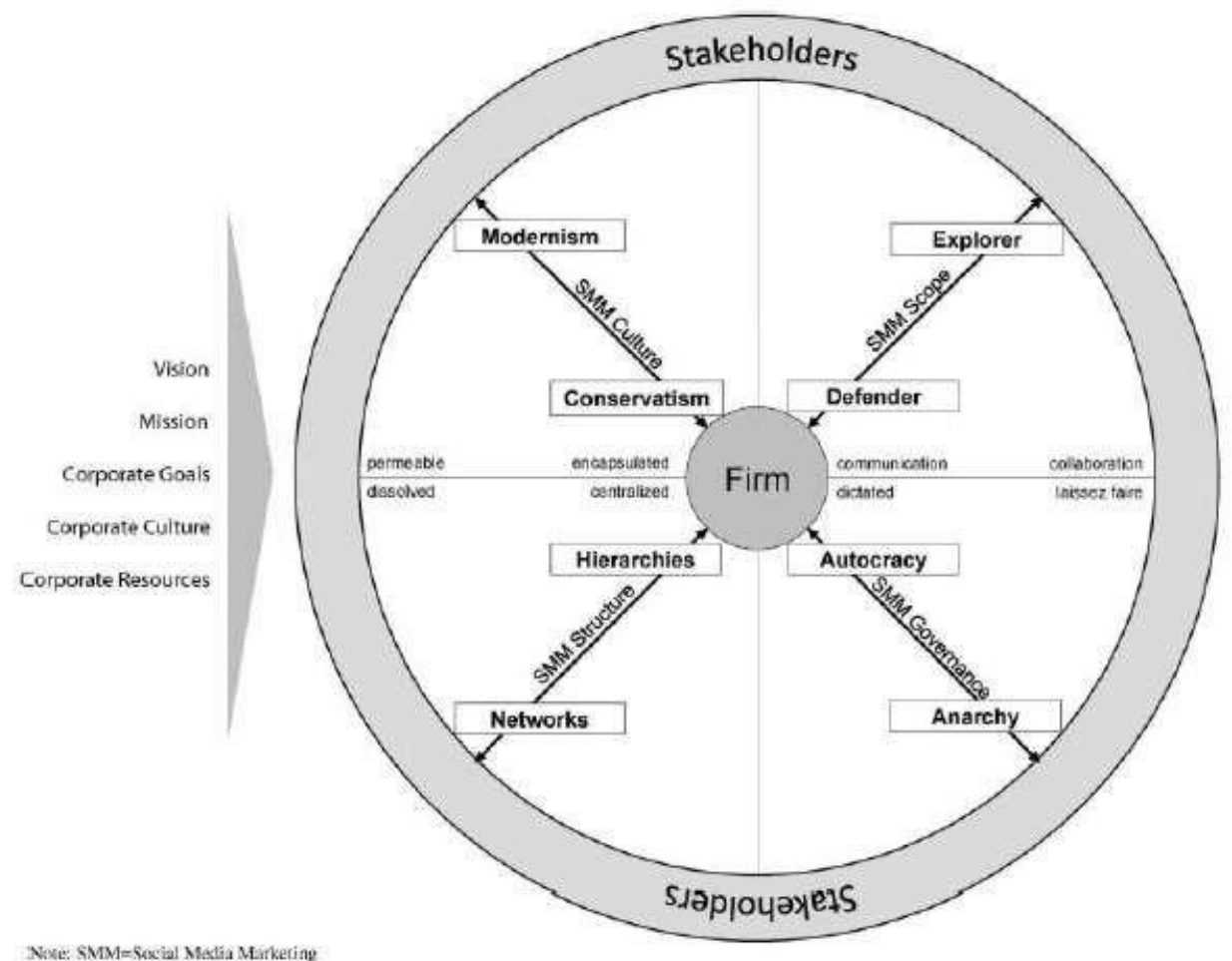

Nove: SMMEsocial Malia Meirkeriag

\section{Gráfico 1. Marco estratégico del marketing de redes sociales}

Defensores y exploradores. Alcance de comercialización

Por un lado, las empresas utilizan el social media marketing como herramienta de comunicación pura para insertar contenido en clientes, la comunidad o empleados. Este enfoque, denominado enfoque de defensa, empleado por los defensores no se recomienda generalmente, por lo general se centra en uno o unos pocos grupos de interés. Por ejemplo, los defensores utilizan el bespassungsfunktion (expresión alemana, traducido en términos generales como "entrar en la funciónentretenimiento"), que se refiere a la práctica actual de la posibilidad de subir contenido divertido o de otro modo entretenido, como una característica del enfoque de defensa. Este enfoque ofrece un marcado contraste con el enfoque explorador, que se centra en el aprovechamiento del potencial de integración, interactivo y de colaboración de la tecnología de las redes sociales. En síntesis, en el enfoque de defensa, los consumidores pueden tratar de comunicarse entre sí y con la empresa a través de los medios sociales, pero reciben respuestas estandarizadas o incluso ninguna respuesta en absoluto (Felix et al., 2016).

Por otra parte, los "exploradores" son compañías que utilizan el social media marketing para crear y mantener relaciones reciprocas con las partes interesadas. Los exploradores se centran en un enfoque de colaboración para los medios de comunicación social de marketing donde se incluye muchos de los grupos de interés, tales como los clientes actuales y potenciales, empleados actuales y potenciales, proveedores o sociedad en general. Adquirir y utilizar la información proporcionada a través de plataformas de medios sociales de cada uno de estos actores se convierte 


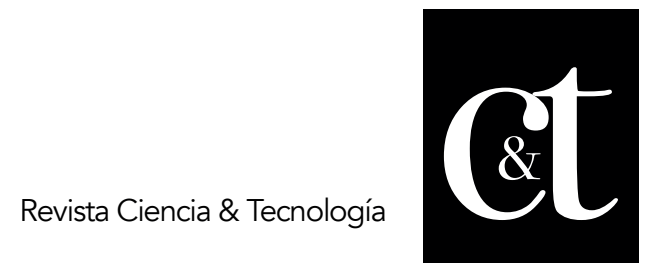

No. 17, 31 de enero de 2018

ISSN impreso: 1390 - 6321

en un factor importante en este enfoque e incluye definir cómo las diferentes partes interesadas pueden cohesionarse en el proceso de creación de valor de. El enfoque explorador, integrado de dos vías comunicación y colaboración que están completamente abiertas, en lugar de limitarse a transmitir información, colaboran en el contexto del enfoque explorador, como la colaboración entre la firma y clientes o entre varios empleados, además se utiliza como una herramienta para la gestión de los interesados. Los medios sociales deberían motivar a la gente para compartir información (Felix et al., 2016).

Cultura del social media marketing. Conservadurismo y modernismo

Existen dos posiciones extremas relacionadas con la cultura de marketing en redes sociales. La primera posición, el conservadurismo, refleja una visión encapsulada, internamente enfocada y con aversión al riesgo del marketing en redes sociales. Sin embargo, se entiende que el marketing exitoso en redes sociales requiere una cultura abierta, permeable, auténtica $y$, a veces, arriesgada. Por lo tanto, una cultura moderna de mercadeo en redes sociales, respaldada por la alta gerencia, puede obligar a las compañías a reevaluar cómo se ejecutan las iniciativas en la organización. Debido a que la cultura organizacional es un determinante relativamente estable de eficacia de la organización (Zheng, Yang, y McLean, 2010), las empresas con una cultura corporativa más tradicional (es decir, incongruentes con la apertura necesaria para la implementación exitosa de marketing en redes sociales) se enfrentan a un desafío difícil. Cuando se los compara con el marketing de medios tradicional, los consumidores pueden tomar el control de muchos aspectos del contenido corporativo publicado en las redes sociales (Labrecque et al., 2013). Por lo tanto, las empresas se ven obligadas a volver a evaluar el nivel de propiedad que dicen mantener con respecto a sus mensajes de marketing en estas plataformas. Además, los especialistas en marketing deberían agradecer la atención de los usuarios sobre el contenido de las redes sociales de la empresa, incluso cuando las interacciones de los consumidores con este contenido no son coherentes con el mensaje previsto de la empresa y producen transformación o incluso deformación de los mensajes.

Estructura de marketing en redes sociales. De las jerarquías a las redes La investigación actual identifica dos posiciones extremas para la estructura de marketing en redes sociales: jerarquías (que representan una estructura de marketing de medios sociales centralizada y concentrada) y redes (es decir, en las que el control del marketing en redes sociales es fragmentado, descentralizado, disuelto y multifuncional). Las jerarquías representan empresas con una gran necesidad de control y una organización cuasi militar.

Por el contrario, la idea de una estructura en red para el marketing en redes sociales implica que las actividades de las redes sociales son una responsabilidad común de todos los empleados, independientemente del departamento al que estén asignados. Esta idea es similar al concepto de gestión de calidad moderna en el que la calidad es el trabajo de todos los empleados de la empresa, no simplemente el ámbito de un "departamento de calidad" (Lai \& Cheng, 2005). Esta perspectiva sugiere que el social media marketing debe ser una función que impregna todos los procesos y departamentos de la organización, y, por lo tanto, un medio de comunicación social, director de marketing o especialista tomarían el papel de la dirección, en lugar de ejecutar, los esfuerzos de comercialización de las redes sociales de una empresa, si 


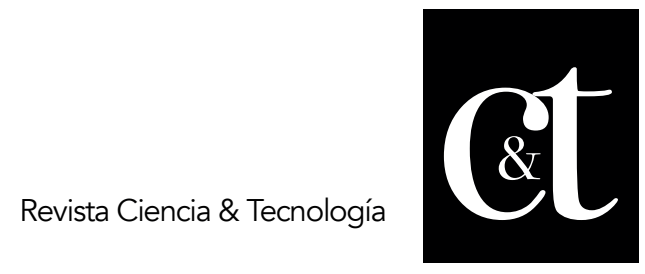

No. 17, 31 de enero de 2018

ISSN impreso: 1390 - 6321

se requieren sus servicios. En el último paso, el representante de redes sociales ya no existe, porque las redes sociales se han incorporado al ADN de la empresa. Por supuesto, esto puede demorar cinco o diez años, pero eventualmente no habrá ningún especialista en redes sociales, ya que se ha incorporado a las subáreas de la empresa.

\section{Autocracia y anarquía}

La cuarta dimensión se refiere a cómo se rige el marketing en redes sociales en la empresa. La importancia de las pautas y políticas para que los empleados determinen quién está facultado en la empresa para proporcionar qué tipo de contenido en qué aplicación de redes sociales. El continuo de posibilidades sugiere dos posiciones extremas denominadas "autocracia" y "anarquía". En la autocracia, un solo departamento centraliza y administra el control de la comunicación en las redes sociales. Por el contrario, la anarquía está representada por una mentalidad de laissez-faire en la que no existen tales reglas y los departamentos / empleados son libres de comunicarse a voluntad en las plataformas de medios sociales. A medida que la cultura y la estructura organizativa se vuelven más abiertas, la definición de pautas claras de redes sociales se vuelve cada vez más importante. Por ejemplo, unas directrices claras son fundamentales para una empresa posicionada en el lado de la red de la estructura de la "estructura de marketing de redes sociales" (es decir, cualquier empleado de cualquier departamento, en lugar de un único individuo o departamento, puede ejecutar marketing en redes sociales) y menos para las empresas con una estructura jerárquica de marketing en redes sociales. La capacitación en redes sociales puede acercar a la empresa al polo "autocracia" de la dimensión de gobernanza del marketing en redes sociales. Sin la capacitación adecuada, la calidad y el profesionalismo del marketing en redes sociales sufrirían.

\section{Discusión}

Este desarrollo se fundamenta en base a la ausencia de un marco integral de marketing estratégico en medios sociales. Una revisión de la literatura similar de marketing en varios enfoques y que guardan relación con los aspectos de marketing estratégico, así como la gestión de relaciones con los clientes son las expuestas por Payne y Frow (2005), así como la organización de marketing de Workman, Homburg, \& Gruner (1998). Como siempre, algunos artículos se refieren a la comercialización estratégica de los medios sociales, y ninguno presentó un marco de medios de comunicación social de marketing integral.

Si bien la investigación existente relacionada con el social media marketing investiga los medios sociales en su mayoría a través de la lente de un problema particular de marketing (por ejemplo, Fong \& Burton, 2008; Kim \& Ko, 2012; Kumar et al., 2016) o con un enfoque en los clientes y la comunicación (Chang et al., 2015), el detalle de este estudio revela cuatro dimensiones generales de marketing de medios sociales, que las empresas deberían abordar al conceptualizar el marketing digital.

El marketing en medios sociales ofrece a las organizaciones una oportunidad de usar los medios sociales para construir relaciones con los clientes, empleados, comunidades, y otros grupos de interés (es decir, cuando actúan como exploradores). Al mismo tiempo, las empresas pueden elegir ver los medios sociales simplemente como otro canal de comunicación por el cual pueden empujar información a los clientes (es decir, cuando actúan como defensores). 


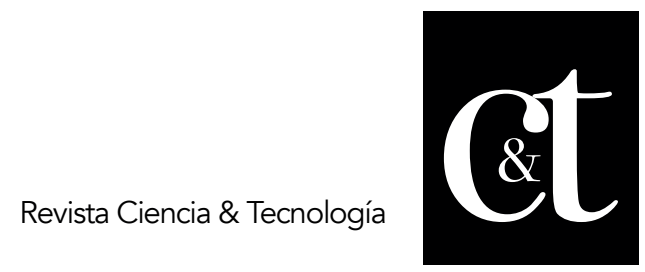

No. 17, 31 de enero de 2018

ISSN impreso: 1390 - 6321

Investigadores de gestión y comportamiento organizacional (Zheng et al., 2010) así como académicos y profesionales de marketing (Deshpandé \& Farley, 2004) reconocen la importancia de la cultura y el clima de la organización para rendimiento financiero y no financiero. El desarrollo actual hace hincapié en la importancia de la cultura de los medios de comunicación social para el mercado. Sociedades con marketing en medios sociales deben reconocer que los interesados puedan tomar el control de los mismos y manipularlos (Labrecque et al., 2013). Por lo tanto, las empresas deben contemplar la cultura encapsulada en medios de comunicación social de marketing (conservadurismo), que proporciona un mayor control de la "marca", y el deseo de los consumidores de conectar y relacionarse de forma eficiente (es decir, el modernismo).

Futuras investigaciones podrían tomar un enfoque diferente al expuesto en este desarrollo. Tomar como referencia para estudios posteriores enfoques cuantitativos ayudarían a identificar el impacto de las diferentes posiciones de cada una de las dimensiones propuestas, los investigadores que decidan tomar como referencia el presente desarrollo no deben pasar por alto los contextos culturales o las dimensiones económicas de la población donde se realice la investigación, así como aspectos organizacionales asociados al clima laboral, el tipo de industria, el tamaño de la misma, los recursos que posee o donde y bajo que parámetros políticos desarrollas sus actividades.

\section{Conclusiones}

Este desarrollo teórico arroja a la luz la naturaleza compleja del marketing estratégico en redes sociales. El marketing en redes sociales, en la práctica, es demasiado complejo para ser administrado y ejecutado exclusivamente por un solo individuo o incluso por un departamento. Las colaboraciones multifuncionales a lo largo de las cuatro dimensiones del marketing en redes sociales son necesarias para navegar con éxito en este dinámico escenario. La persona encargada del proceso de marketing en redes sociales de una organización debe replantear su forma de trabajo individual para un mejor desempeño, y una posterior obtención de resultados favorables para la industria en la cual presta sus servicios.

\section{Referencias Bibliográficas}

Ashley, C., \& Tuten, T. (2015). Creative strategies in social media marketing: An exploratory study of branded social content and consumer engagement. Psychology \& Marketing, 32(1), 15-27.

Asmussen, B., Harridge-March, S., Occhiocupo, N., \& Farquhar, J. (2013). The multilayered nature of the internet-based democratization of brand management. Journal of Business Research, 66(9), 1473-1483.

Baird, C.H. \& Parasnis, G. (2013). From social media to social customer relationship management. IEEE Engineering Management Review 41(3):48-55.

Bernoff, J., \& Li, C. (2008). Harnessing the power of the oh-so-social web. MIT Sloan Management Review, 49(3), 36-42.

Bianchi, C., \& Andrews, L. (2015). Investigating marketing managers' perspectives on social media in Chile. Journal of Business Research, 68(12), 2552-2559.

Brunner-Sperdin, A., Scholl-Grissemann, U.S. y Stokburger-Sauer, N.E. (2014). El 


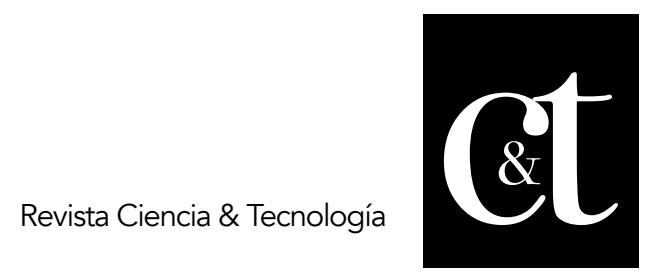

No. 17, 31 de enero de 2018

ISSN impreso: 1390 - 6321

perti-Vance página web de la percepción holística. Cómo sentido de decisiones y las señales de exploración guía las emociones y los comportamientos de los consumidores. Journal of Business Research, 67(12), 2515-2522.

Canhoto, A. I., \& Clark, M. (2013). Customer service 140 characters at a time: The users' perspective. Journal of Marketing Management, 29(5-6), 522-544.

Chang, Y. T., Yu, H., \& Lu, H. P. (2015). Persuasive messages, popularity cohesion, and message diffusion in social media marketing. Journal of Business Research, 68(4), 777-782.

De Swaan Arons, M., van den Driest, F., \& Weed, K. (2014). The Ultimate Marketing Machine. Harvard Business Review, 92 (7), 54-63.

Deshpandé, R., \& Farley, J. U. (2004). Organizational culture, market orientation, innova-tiveness, and firm performance: An international research odyssey. International Journal of Research in Marketing, 21(1), 3-22.

Divol, R., Edelman, D., \& Sarrazin, H. (2012). Demystifying social media. McKinsey Quarterly, 2(12), 66-77.

Eyrich, N., Padman, M.L., \& Sweetser, K.D. (2008). PR practitioners' use of social media tools and communication technology. Public Relations Review 34 (2008), 412-414

Felix, R., Rauschnabel, P., \& Hinsch, C. (2016). Elements of strategic social media marketing: A holistic framework, Journal of Business Research, 70.

Fong, J., \& Burton, S. (2008). A cross-cultural comparison of electronic word-ofmouth and country-of-origin effects. Journal of Business Research, 61(3), 233242.

Fournier, S., \& Avery, J. (2011). The uninvited brand. Business Horizons, 54(3), 193207.

Gebauer, J., Füller, J., \& Pezzei, R. (2013). The dark and the bright side of cocreation: Triggers of member behavior in online innovation communities. Journal of Business Research, 66(9), 1516-1527.

Hennig-Thurau, T., Malthouse, E. C., Friege, C., Gensler, S., Lobschat, L., Rangaswamy, A., \& Skiera, B. (2010). The impact of new media on customer relationships. Journal of Service Research, 13(3), 311-330.

Kietzmann, J., Hermkens, K., McCarthy, I., \& Silvestre, B. (2011). Social media? Get serious! Understanding the functional building blocks of social media. Business Horizons, 54.

Kim, A.J. \& Ko, E. (2012). Do social media marketing activities enhance customer equity? An empirical study of luxury fashion brand. Journal of Business Research 65(10).

Kumar, A., Bezawada, R., Rishika, R., Janakiraman, R., \& Kannan, P. K. (2016). From social to sale: The effects of firm-generated content in social media on consumer behavior. Journal of Marketing, 80(1), 7-25.

Linke, A. \& Zerfass, A. (2013). Social Media Governance: Regulatory frameworks for successful online communications. Journal of Communication Management $17(3): 270-286$. 


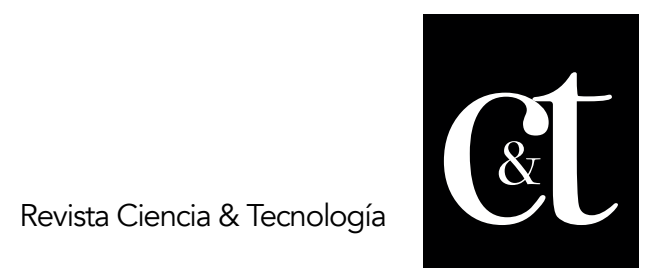

No. 17, 31 de enero de 2018

ISSN impreso: 1390 - 6321

Labrecque, L., vor dem Esche, J., Mathwick, C., Novak, T., \& Hofacker, C. F. (2013). Consumer power: Evolution in the digital age. Journal of Interactive Marketing, 27(4), 257-269.

Lai, K. \& Cheng, T.C.E. (2005). Effects of quality management and marketing on organizational performance. Journal of Business Research 58(4):446-456.

Naylor, R. W., Lamberton, C. P., \& West, P. M. (2012). Beyond the "like" button: The impact of mere virtual presence on brand evaluations and purchase intentions in social media settings. Journal of Marketing, 76(6), 105-120.

Olson, E. M., Slater, S. F., \& Hult, G. T. M. (2005). The performance implications of fit among business strategy, marketing organization structure, and strategic behavior. Journal of Marketing, 69(3), 49-65.

Payne, A., \& Frow, P. (2005). A strategic framework for customer relationship management. Journal of Marketing, 69(4), 167-176.

Quinton, S. (2013). El paradigma de la marca comunitaria: Una respuesta a la gestión de la marca dilema en la era digital. Diario de Dirección de Marketing, 29 (7/8), 912-932.

Relling, M., Schnittka, O., Sattler, H., \& Johnen, M. (2016). Each can help or hurt: Negative and positive word of mouth in social network 19 brand communities. International Journal of Research in Marketing, 33(1), 42-58.

Rokka, J., Karlsson, K., \& Tienari, J. (2014). Balancing acts: Managing employees and reputation in social media. Journal of Marketing Management, 30(7/8), 802827.

Schultz, D. E., \& Peltier, J. (2013). Social media's slippery slope: Challenges, opportunities and future research directions. Journal of Research in Interactive Marketing, 7(2), 86-99.

Schweidel, D. A., \& Moe, W. W. (2014). Listening in on social media: A joint model of sentiment and venue format choice. Journal of Marketing Research, 51(4), $387-402$.

Sivertzen, A., Nilsen, E., \& Olafsen, A. (2013). Employer branding: Employer attractiveness and the use of social media. Journal of Product \& Brand Management, 22(7), 473-483.

Toca, C.E. (2013). El marketing al servicio de las organizaciones del tercer sector. Estudios Gerenciales 29 (2013), 386-395.

Trainor, K. J., Andzulis, J. M., Rapp, A., \& Agnihotri, R. (2014). Social media technology usage and customer relationship performance: A capabilities-based examination of social CRM. Journal of Business Research, 67(6), 1201-1208.

Thompson, C. J., \& Coskuner-Balli, G. (2007). Countervailing market responses to corporate co-optation and the ideological recruitment of consumption communities. Journal of Consumer Research, (34)2, 135-152.

Workman, J.P., Homburg, C., \& Gruner, K. (1998). Organización de marketing: Una integración marco de dimensiones y determinantes. Journal of Marketing, 62 (3), 21-41.

Yadav, M. S., \& Pavlou, P. A. (2014). Marketing in computer-mediated environments: 


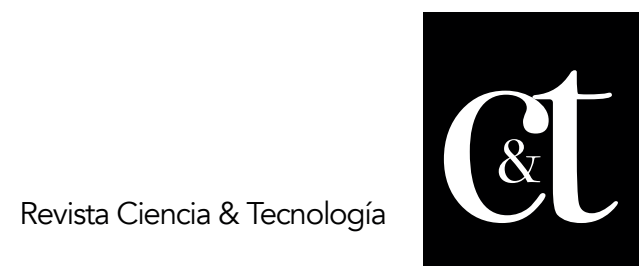

No. 17, 31 de enero de 2018

ISSN impreso: 1390 - 6321

Research synthesis and new directions. Journal of Marketing, 78(1), 20-40.

Zheng, W., Yang, B., \& McLean, G. N. (2010). Linking organizational culture, structure, strategy, and organizational effectiveness: Mediating role of knowledge management. Journal of Business Research, 63(7), 763-771. 